“ (C) 2018 IEEE. Personal use of this material is permitted. Permission from IEEE must be obtained for all other uses, in any current or future media, including

reprinting/republishing this material for advertising or promotional purposes, creating new collective works, for resale or redistribution to servers or lists, or reuse of any copyrighted component of this work in other works." 


\title{
Low-Complexity Digital Modem Implementation for High-Speed Point-to-Point Wireless Communications
}

\author{
Hao Zhang, Xiaojing Huang and Y. Jay Guo \\ Global Big Data Technologies Centre \\ University of Technology Sydney, Australia \\ Emails: \{Hao.Zhang, Xiaojing.Huang, Jay.Guo\}@uts.edu.au
}

\begin{abstract}
A low-complexity digital modem is presented in this paper for achieving high-speed and wideband point-topoint (P2P) wireless communications. By combining multiple functionalities into the transmitter and receiver filters, the signal processing complexity in the digital baseband can be significantly reduced. The structures and the implementation using field programmable gate array (FPGA) for the transmitter and receiver filters are described in details. Pre-equalization for reducing the impact of practical channel frequency response can be easily incorporated into the transmitter filter structure. The experimental test results using a 20 Gigabits per second (Gbps) digital modem prototype demonstrate the satisfactory performance with low FPGA resource usage.
\end{abstract}

Keywords-Low-Complexity, High-Speed, Wideband, Wireless Backhaul, and FPGA Implementation.

\section{INTRODUCTION}

High-speed point-to-point (P2P) wireless links play a pivotal role in wireless communication systems by handling the aggregation and distribution of various data flows such as voice, video, Internet, and other data sources. They provide cost-effective ways to offer tens or even hundreds of Gbps data rates and hence can be used as backhaul links in cellular networks, such as the fifth generation (5G) systems [1] and aerial backbones in space and terrestrial integrated networks.

A typical backhaul system consists of digital baseband, intermediate frequency (IF) and/or radio frequency (RF) modules. Any imperfection in various filters of these modules as well as wireless multipath fading channel will introduce intersymbol interference (ISI) at the receiver. As the transmission rate increases, ISI becomes more serious and deteriorates the date detection performance significantly. The channel equalization is commonly used at the receiver side for coping with ISI. At the transmitter side, pre-equalization is also an effective and low-complexity way to be implemented by using a predefined pulse shaping filter based on off-line channel sounding. In this way, the imperfection of the RF and/or IF modules can be pre-compensated and the equalization complexity at receiver side can be substantially reduced, especially for wideband systems.

In-phase and Quadrature-phase (I/Q) imbalance is another significant factor for a wideband wireless backhaul system with I/Q modulation architecture where a data symbol is modulated onto (or demodulated from) an IF or RF carrier via two separate in-phase (I) and quadrature (Q) channels. The signal will be distorted if there is any difference between I and $\mathrm{Q}$ channel characteristics. There are a number of methods for I/Q imbalance compensation in digital domain and/or analog domain. Most of those methods deal with I/Q imbalance compensation at the receiver side independently.

In order to suppress the effect of ISI and reduce the I/Q imbalance, significant researches have been conducted and a number of techniques can be found in the literature. For example, a complex infinite impulse response (IIR) filter with digital pre-equalization is proposed in [2]. This preequalization can deal with various linear distortions as well as the channel distortion. A two stage iteration-based algorithm is proposed in [3], which is used at transmitter to calculate a pre-filter. The hardware implementation of the blind matched filter receiver is described in [4], by which the matrix inversion and matrix multiplication operations are replaced by a simple recursive algorithm for the filter design. The channel identification and equalization performance achieve almost equal mean squared error (MSE) and bit error rate (BER) levels compared to those of the conventional receivers. In [5], a simple non-matched receiver is developed for an orthogonal pulse shape modulation scheme in ultra-wideband (UWB) communication system. An iterative decision feedback receiver for single carrier frequency domain equalization (SCFDE) is proposed in [6] to compensate the I/Q imbalance. Compared with conventional linear methods, this receiver can significantly improve the performance of SC-FDE system under I/Q imbalance.

However, in all of the above mentioned techniques, the channel equalization and I/Q imbalance compensation are performed separately. With these conventional techniques, it is very hard to achieve low-complexity implementation for wideband systems which demand significant signal processing resources. In terms of complexity reduction, it is necessary to optimize each digital process (such as transmitter filtering, channel estimation, receiver filtering and detection) so that all signal processing functionalities can be implemented in FPGA with minimum power and volume. In doing so, any wide wordlength operation for large matrix which has more than three dimensions or recursion in the algorithm should be avoided. On the other hand, high-speed P2P wireless systems usually 
have much wider bandwidth such as multiple $\mathrm{GHz}$ in order to achieve the tens or even hundreds of Gbps data rate. With such wide bandwidth, the IF and/or RF modules are hard to build and some undesirable performance such as significant ripple in the pass band and frequency-dependent IQ imbalance would be demonstrated in practical hardware.

In this paper, the low-complexity digital modem implementation for high-speed wideband wireless communications is presented focusing on efficient transmitter and receiver filters designs respectively. Each of these filters combines multiple signal processing tasks together. To be specific, the transmitter filter performs both sample rate conversion (SRC) and pulse shaping with the capability of pre-equalization. The receiver filter performs SRC, channel equalization, and I/Q mismatch compensation at the same time. Therefore, the overall implementation complexity is significantly reduced as compared to conventional designs. Adopting a 20 Gbps single carrier system as an example, how to implement the transmitter and receiver filters in Xilinx Virtex 7-690T FPGA is described in details. Total FPGA resource usage together with optical Ethernet interface, data symbol mapping/demapping, and low density parity check (LDPC) encoding/decoding is provided. Real-time experimental test results are also given. To the authors' knowledge, similar work that produces such high data rate over such wide bandwidth with such low implementation complexity has not been found in the literature.

The rest of the paper is organized as follows. In Section II, system descriptions on the digital modem architecture as well as transmitter and receiver structures are presented. In Section III, FPGA implementation for transmitter and receiver filters is described in details and resource usage is provided. Test setup and experimental results are shown in Section IV. Finally, Section V concludes this paper.

\section{SySTEM DESCRIPTION}

\section{A. Digital Baseband and IF Architecture}

The high-speed digital modem presented in this paper consists of a digital baseband platform and an IF module. Depending on different applications, this digital modem can be connected to different RF frontends to become a Ka band, millimeter wave, and/or terra Hertz ( $\mathrm{THz})$ communication systems. Fig. 1 shows the system architecture with the digital baseband platform and IF module. The digital baseband platform is composed of two FPGAs, each capable of processing $10 \mathrm{Gbps}$ data rate, and an IF module for transmitter and receiver respectively. When fully operated, the digital modem can transmit and receive Ethernet traffic at 20 Gbps data rate simultaneously. Between the FPGAs and the IF module, the high-speed digital-to-analog converters (DACs) and analog-todigital converters (ADCs) are connected, with sampling rate 2.5 Gsps.

The functionality of the IF module is to up-convert the I/Q modulated baseband signals (4 channels) generated by the baseband platform to the $15.65 \mathrm{GHz}$ IF band at the transmitter, and down-convert the IF signal to 4 channel baseband signals which are then received by the baseband platform. A 15.65

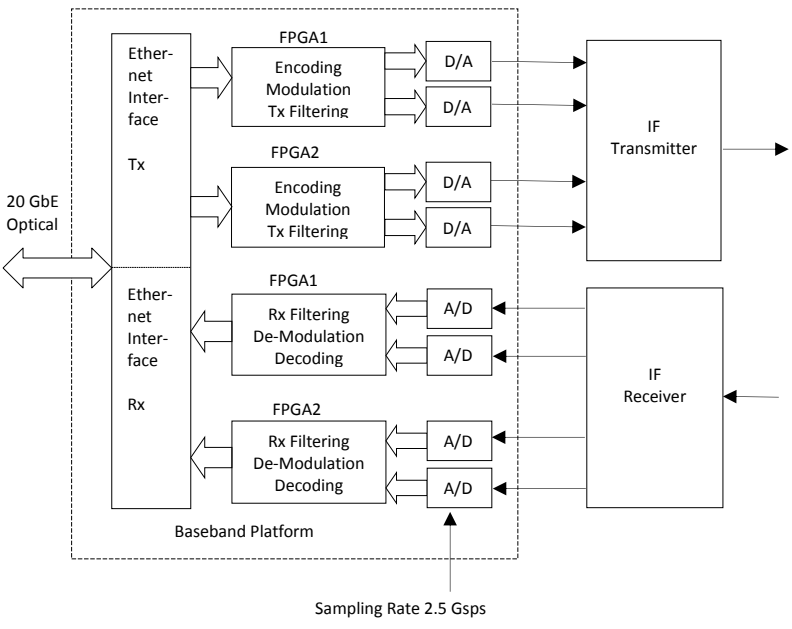

Fig. 1. The system architecture.

$\mathrm{GHz}$ pilot frequency is also added at the transmitter for carrier frequency tracking.

The transmitter filter and receiver filter play important roles in the digital modem, which are the focuses in this paper.

\section{B. Transmitter Filter Structure}

The transmitter filters combine the SRC and pulse shaping together. The SRC is implemented by using the polyphase filter bank approach. In this system design, the symbol rate after modulation (also called data symbol mapping) is 1.875 Giga-samples per second (Gsps) and the signal sampling rate at DACs is 2.5 Gsps. That is, in every 3 symbols duration, there will be 4 samples generated. Since the conversion ratio is $4 / 3$, the number of filters in the filter bank is three. Each filter is a root-raised-cosine (RRC) pulse shaping filter sampled at $2.5 \mathrm{Gsps}$ but with different time offsets. The structure of the filter bank is shown in Fig. 2.

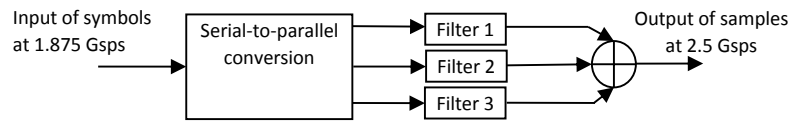

Fig. 2. Structure of transmitter filter.

\section{Receiver Filter Structure}

The receiver filters simultaneously perform the I/Q imbalance compensation, channel equalization, and SRC functions. The block diagram of the receiver filter bank and the structure of each polyphase filter are shown in Fig. 3 and Fig. 4 respectively. A polyphase filter has two parts which are used for filtering the real part and imaginary part of the received signal at 2.5 Gsps respectively. After channel and I/Q mismatch estimation, the filter coefficients of these two parts can be calculated in real-time in FPGA. 


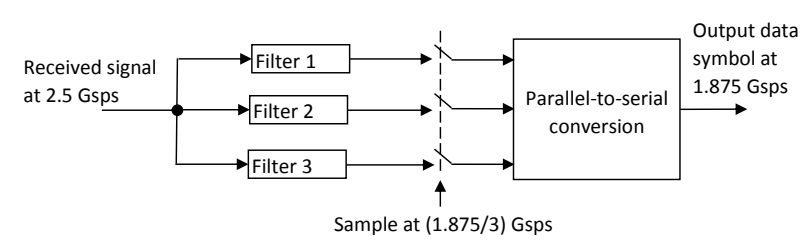

Fig. 3. Structure of receiver filter.

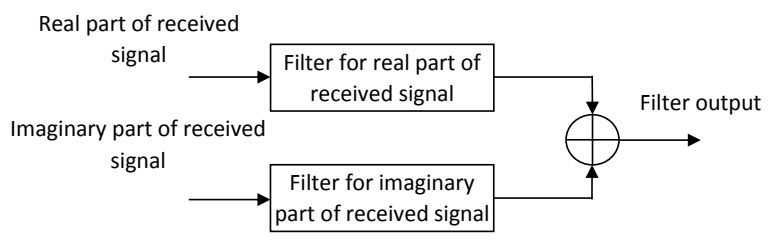

Fig. 4. Structure of polyphase filter.

\section{FPGA IMPLEMENTATION}

\section{A. Transmitter Filter}

The design of transmitter filters without or with preequalization affects the complexity of channel equalization at the receiver side. In our design, each transmitter filter is an RRC pulse shaping filter with pre-equalization sampled at 2.5 Gsps with different time offset. When pre-equalization is used, coefficients of filters vary with the condition of the IF module. Therefore, filter coefficients can be configured when the FPGA bit-file is generated after channel sounding to determine the channel response of the IF module. In order to be used for wideband system in practice, the length of transmitter filters can not be very small so that the length is chosen as 32 in this design. With different time offsets, each transmitter filter output sample is generated by 24 addition operations with 12 bit width per addition operation, and eight samples are generated in each FPGA high-speed clock period (312.5 $\mathrm{MHz}$ ). Considering the filter coefficient configurability and the huge number of addition operations with large word-width, the efficient and effective architecture of transmitter filter is shown in Fig. 5.

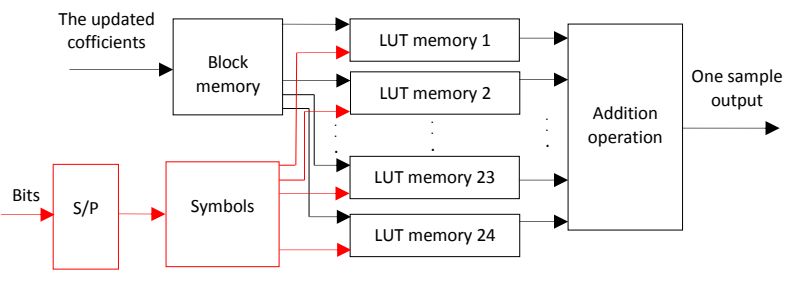

Fig. 5. Structure of Tx filter for one sample.

After setting up the digital modem in the IF loopback mode, a special training sequence is sent from the digital baseband platform through the IF module and received by the digital baseband platform. Coefficients for transmitter filters can be calculated by the captured data from the digital receiver. The updated coefficients for different conditions of the IF module can be uploaded into the block memory. The size of coefficients is around $24 \mathrm{Kbits}$. Due to the sufficient block memories in FPGA [7], it is reasonable to use $1 \times 36$ Kbits block memory for storing these coefficients. Following the designed structure of the transmitter filters, one output sample is generated with 24 time offsets in one FPGA clock period. Therefore, coefficients stored in the block memory are divided into 24 small groups, and the size of each group is 1 Kbits. Considering the small size of each small group of coefficients and the minimum of 18 Kbits for each block memory, each small group of coefficients is stored in lookup table (LUT) memory rather than the block memory. At the same time, the structure of LUT memory is optimized for highspeed clock frequency when routing all cells in FPGA. Once coefficients are downloaded into 24 LUT memories, samples can be generated following the inputs of symbols which are generated from serial-to-parallel converter. The data outputs from 24 LUT memories are added together to generate one output sample. The details of the adder for long bit-width signal are described in [8].

\section{B. Receiver Filter}

As we know, the length of receiver filters affects the performance and complexity when implementing the system in practice. Considering these two factors, this design adopts length 54 receiver filters. From Fig. 3 and Fig. 4, we can easily find that a large number of multiplications and additions are necessary for the long receiver filters. Multiplications can be implemented by DSP48 in FPGA [7]. For the addition operations, the length of bits output from DSP48 should be decided for the given precision. In order to achieve good performance, long bit width should be adopted. However, at the same time, the complexity will be increased with the increasing length of bits. Therefore, an appropriate strategy is required to deal with the large number of additions with wide bits. Fig. 6 shows the effective structure of an addition tree.

According to the length of receiver filters, there are 54 multiplications for generating 54 data outputs which should be added together. However, we can not complete all adders in one clock period (312 MHz). Therefore, we divide all adders

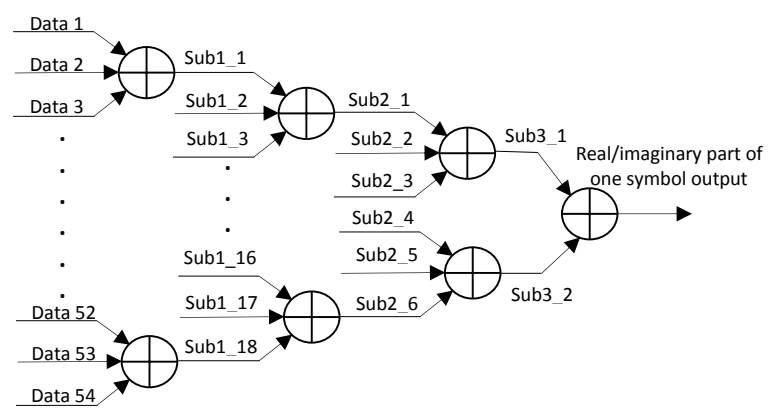

Fig. 6. Structure of addition tree for the real/imaginary part of one symbol. 
into four levels. Considering the usage rate and the timing of system clock, we select three data additions in one clock period. In this way, the same resources are needed as that for adding two data in one clock period. At the input of the first level, the length of data bits after multiplications should be long enough to satisfy the given precision requirement. However, after adding each level, the sum of each adder increases. Therefore, one bit can be reduced at the input of next level. With this design, the lengths of input data are 13, 12, 11 and 10 bits for each level respectively. In one clock period, six symbols should be generated and each symbol consits of real and imaginary parts. With this addition tree structure, it is effective to keep the precision while reducing the resource usage.

\section{Implementation Results}

In addition to the above described transmitter and receiver filters, the digital modem also consists of other necessary signal processing modules. At the transmitter side, there are encoding, modulation, transmitter filters and DAC interface modules. At the receiver side, there are synchronization, channel estimation, receive filters, demodulation, decoding and ADC interface modules. Between the digital modem physical (PHY) layer and the network layer, the $20 \mathrm{Gbps}$ transmitter and receiver fiber interfaces are also implemented. These modules are implemented to deal with high throughput for the wideband system, each being optimized in the FPGA. The resource uasge for the two filters and the whole system is shown in Table I.

The powerful device - Virtex7-690T produced by Xilinx is used in our digital baseband platform. From Table I, we can see that resource usage of some typical cells including LUTs, slice registers, block RAMs, multipliers are reasonable for the whole system compared with the total resources in this device. The two filters only occupy a small proportion of the total available resources. The reserved resources can be used for other modules in the whole digital modem.

TABLE I

FPGA USAGE OF TRANSMITTER/RECEIVER FILTERS AND THE WHOLE SYSTEM

\begin{tabular}{|c|c|c|c|c|}
\hline Module Name & Slice LUTs & $\begin{array}{c}\text { Slice } \\
\text { Registers }\end{array}$ & $\begin{array}{c}\text { Block } \\
\text { RAMs }\end{array}$ & Multipliers \\
\hline TX Filter & 11200 & 15400 & 8 & 0 \\
\hline RX Filter & 18600 & 69000 & 12 & 1344 \\
\hline $\begin{array}{c}\text { The Whole } \\
\text { System }\end{array}$ & 245800 & 368600 & 590 & 2220 \\
\hline $\begin{array}{c}\text { Total } \\
\text { Number }\end{array}$ & 433200 & 866400 & 1470 & 3600 \\
\hline $\begin{array}{c}\text { Usage Rate of } \\
\text { Two Filters }\end{array}$ & $6.9 \%$ & $9.7 \%$ & $1.4 \%$ & $37.3 \%$ \\
\hline $\begin{array}{c}\text { Usage Rate of } \\
\text { System }\end{array}$ & $56.7 \%$ & $42.5 \%$ & $40 \%$ & $61.7 \%$ \\
\hline
\end{tabular}

\section{Test Setup And Experimental Results}

\section{A. Test Setup}

The digital modem is composed of two FPGA platforms, each capable of transmitting and receiving two channels of baseband I/Q signals. Each FPGA platform is connected to a control PC via universal serial bus (USB) cable for monitoring and configuring the state of FPGA. The IF transmitter and receiver are connected directly at IF frequency via coaxial cable. A noise generator is used to generate additive Gaussian noise with on/off switch controlled by a spectrum analyser. A cascade of two wideband amplifiers is used to amplify the noise to sufficient power level. Two attenuators (with $1 \mathrm{~dB}$ step and $10 \mathrm{~dB}$ step respectively) are used to control the noise level and a switch driver is connected to them to set the attenuation manually. The spectrum analyser is also used to monitor the transmitted signal power as well as measure both noise and signal powers.

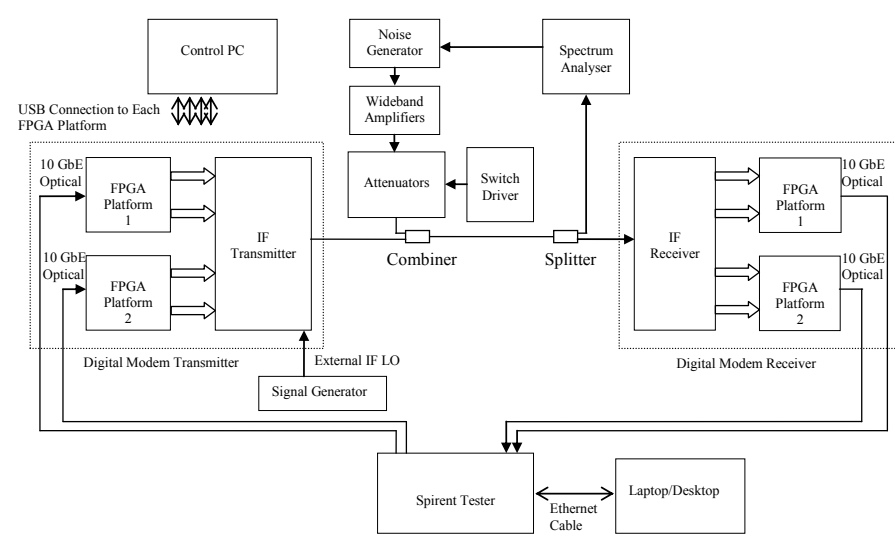

Fig. 7. Structure of test setup.

At the transmitter side, two $10 \mathrm{Gbps}$ Ethernet traffic streams generated by the Spirent tester [9] are pseudo-random bit sequences with 128 byte packet size. At the receiver side, the two received 10 Gbps Ethernet traffic streams are fed back to the Spirent tester, and the BER result can be recorded from the user interface window of Spirent tester. Fig. 7 shows the block diagram of the test setup. A picture of the $20 \mathrm{Gbps}$ digital modem prototype hardware is shown in Fig. 8.

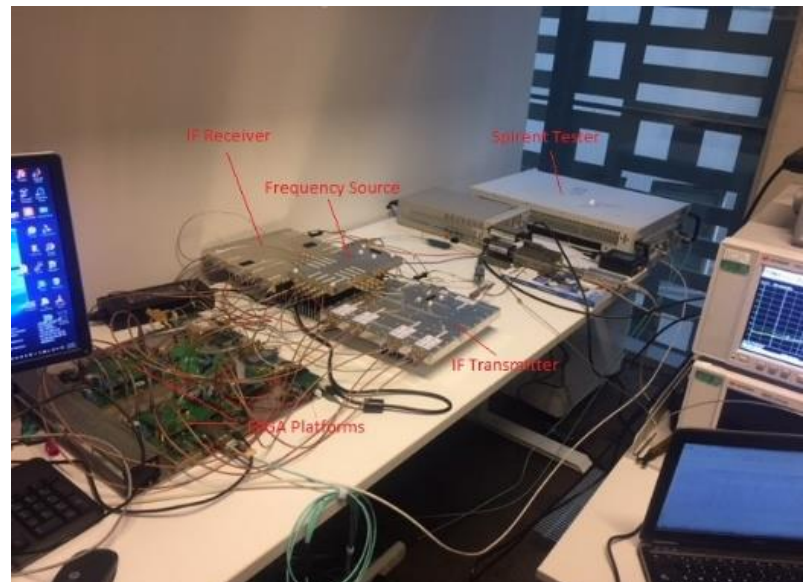

Fig. 8. Picture of $20 \mathrm{Gbps}$ digital modem and test setup. 


\section{B. Experimental Results}

There are four $2.5 \mathrm{GHz}$ channels in the whole system. For simplicity, we just show the result from one typical channel. Other channels produce very similar results.

Before performing real-time system loopback test, the DACs and ADCs should be calibrated first. Due to the baseband I/Q modulation architecture, any difference in terms of delay, phase, and amplitude will introduce I/Q imbalance. After DACs and ADCs calibration, the transmitted signal can be looped back via direct DACs and ADCs connection. The error vector magnitude (EVM) of the constellation is $4.05 \%$ without pre-equalization. From this result, we can make sure that the signal processing produces satisfactory performance for the 16QAM demodulation without IF module.

The test for obtaining the channel frequency response of each channel is performed by transmitting a number of discrete tones throughout the entire bandwidth of each channel. The frequencies of the discrete tones are selected such that their image frequencies appear in-between two original tones, resulting in a shaded area below the frequency response envelope to show the I/Q imbalance.

The overall channel frequency response including both transmitter and receiver for each channel can be obtained in digital baseband at the receiver side. Fig. 9 shows the results calculated using Matlab software after uploading the test data from the digital platform. We see that the channel frequency response fluctuates significantly in the bandwidth of $2.5 \mathrm{GHz}$. The fluctuated range is around $9 \mathrm{~dB}$. At the same time, the image components of the IF module are also quite severe. This poses significant challenges to the signal processing for recover the transmitted data information.

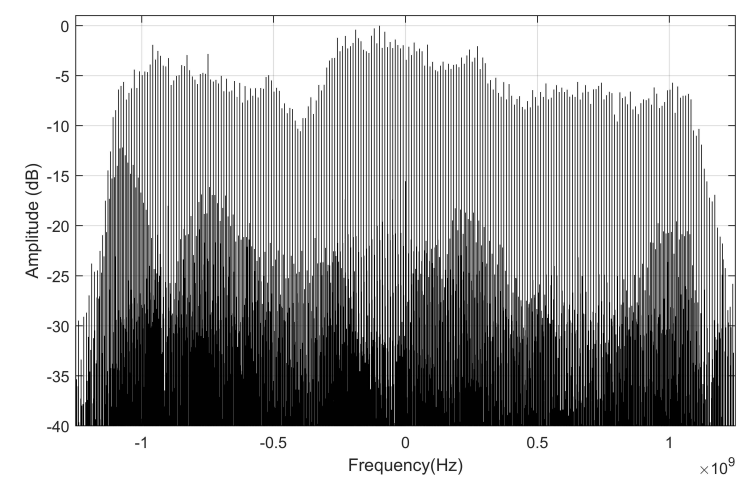

Fig. 9. Frequency response of one IF channel.

Considering the undesirable performance of the IF hardware for the wideband system and in order to reduce the complexity of the whole system as much as possible, an effective way to deal with the significant fluctuation in the channel frequency responses is channel pre-equalization. Fig. 10 shows transmitter filters without pre-equalization. The filters only have the real part without considering the influence from IF module. However, after pre-equalization, transmitter filters have changed a lot. Fig. 11 shows the real part and imaginary part of the transmitter filters with pre-equalization. From this result, we can see that the characters of the signal to be transmitted via the IF module have changed significantly after pre-equalization.

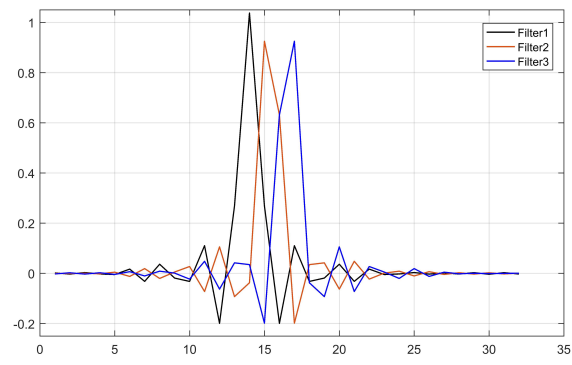

Fig. 10. Tx filters without pre-equalization.

At the receiver side, the characteristics of the channel can be shown from receiver filters. Fig. 12 shows real part of receiver filters without and with pre-equalization (considering the small value of imaginary part, it is not necessary to show imaginary part). Considering the limited resource in the FPGA, we can only use the receiver filters with length 54 . However, for the filters without pre-equalization, the channel response is very long and hence the 54 length filters can not achieve the required performance. For filters with pre-equalization, we can see that the channel impulse is short and concentrated. Even we use a shorter length of the filers after pre-equalization, satisfactory performance is still achieved.

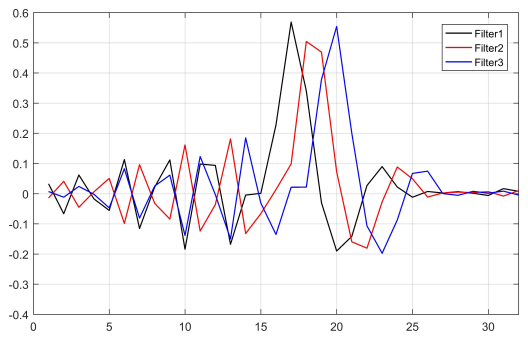

(a)

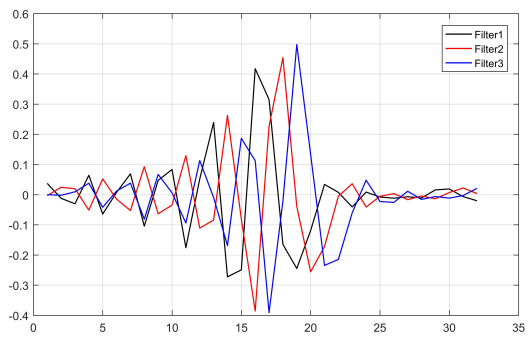

(b)

Fig. 11. Tx filters with pre-equalization of (a) real part and (b) imaginary part.

Fig. 13 shows the comparison between the 16QAM constellations without and with pre-equalization for the selected IF channel. We see that the EVM is significantly improved after 


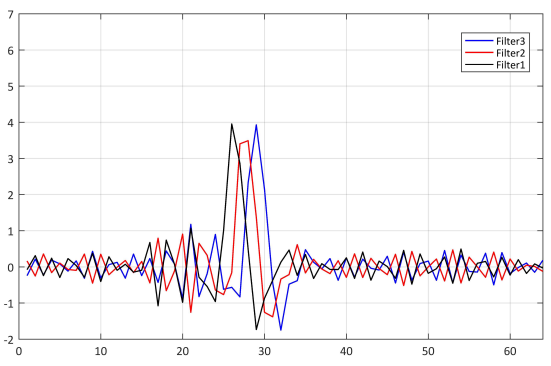

(a)

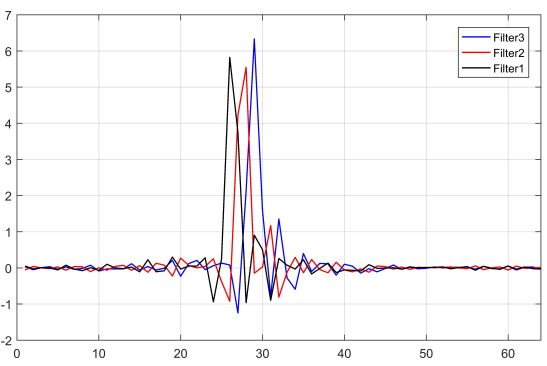

(b)

Fig. 12. Real parts of Rx filters (a) without pre-equalization and (b) with pre-equalization.

pre-equalization. After testing plenty of data, EVMs calculated for the selected channel without pre-equalization and with preequalization are $16.3 \%$ and $10.3 \%$ respectively.

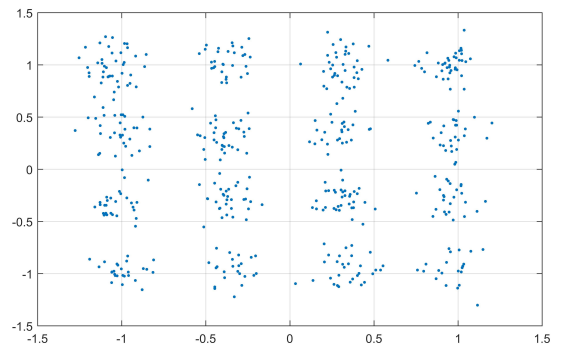

(a)

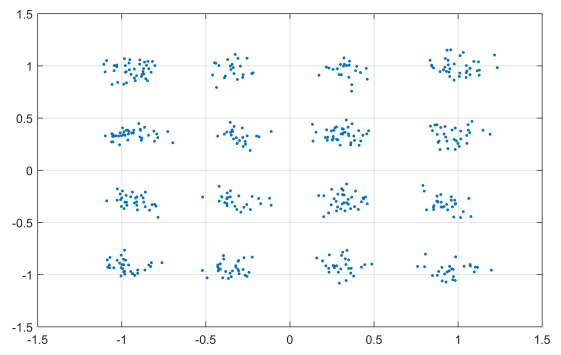

(b)

Fig. 13. Constellations (a) without pre-equalization and (b) with preequalization .

From the above results, we see that pre-equalization is an effective way to reduce the influence of the IF module in the wideband system. After using pre-equalization by updating the transmitter filter coefficients, a huge number of data with realtime Ethernet traffic are tested and the resulting BER versus $\mathrm{Eb} / \mathrm{N} 0$ curve is shown in Fig.14. From this figure, we can see that excellent performance is achieved for this wideband system.

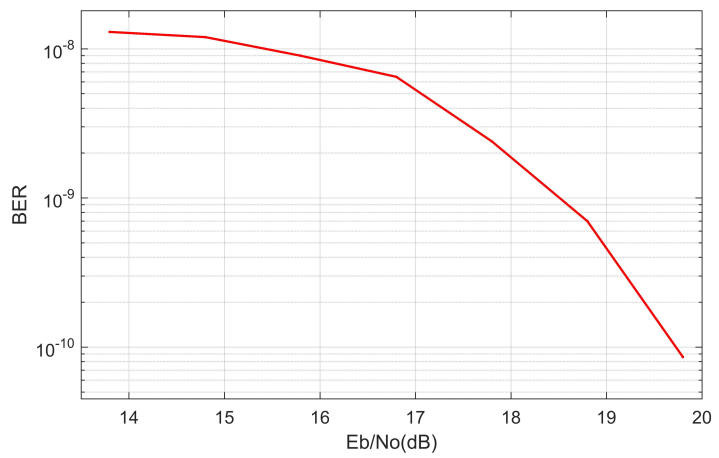

Fig. 14. BER for the selected channel.

\section{CONCLUSION}

In this paper, the low-complexity digital modem implementation for high-speed and wideband system is presented. Optimized architectures are designed for transmitter and receiver filters which can be implemented with low resource usage on the FPGA. The experimental test results using digital and IF hardware prototype verify the excellent performance of the low-complexity designs. We also show that pre-equalization significantly improves the EVM for the 16QAM demodulation with the practical wideband IF module. Adopting the presented filters design and implementation, high-speed wideband wireless communications can be achieved with significant lowcomplexity.

\section{REFERENCES}

[1] J. G. Andrews, S. Buzzi, W. Choi, S. V. Hanly, A. Lozano, A. C. K. Soong, and J. C. Zhang, "What will 5G be?" IEEE Journal on Selected Areas in Communications, vol. 32, no. 6, pp. 1065-1082, June 2014.

[2] H. N. Kim, W. J. Kim, Y. S. Lee, J. H. Seo, S. I. Park, and S. C. Kim, "An adaptive IIR pre-equalizer for terrestrial DTV transmitters," IEEE Transactions on Broadcasting, vol. 53, no. 1, pp. 120-126, March 2007.

[3] S. Alizadeh, H. K. Bizaki, and M. Okhovvat, "Effect of channel estimation error on performance of time reversal-UWB communication system and its compensation by pre-filter," IET Communications, vol. 6, no. 12, pp. 1781-1794, August 2012.

[4] A. Coskun and I. Kale, "All-adaptive blind matched filtering for the equalization and identification of multipath channels - a practical approach," IEEE Transactions on Circuits and Systems, vol. 60, no. 1, pp. 232-242, Jan 2013.

[5] S. Sharma and V. Bhatia, "Performance analysis of filtered PSM signal using non-matched receiver for UWB communication," in 2016 IEEE International Conference on Advanced Networks and Telecommunications Systems (ANTS), Bangalore, India, 2016.

[6] X. Zhang, H. Li, W. Liu, and J. Qiao, "Iterative IQ imbalance compensation receiver for single carrier transmission," IEEE Transactions on Vehicular Technology, vol. 66, no. 9, pp. 8238-8248, Sept 2017.

[7] Xilinx, "FPGA Family," 2010. [Online]. Available: https://www.xilinx. $\mathrm{com} /$ support/documentation/data_sheets/ds180_7Series_Overview.pdf

[8] H. Zhang, X. Huang, and Y. J. Guo, "A 20 Gbps digital modem for high speed wireless backhaul applications," in 2017 IEEE 85th Vehicular Technology Conference (VTC Spring), Sydney, Australia, 2017, pp. 1-5.

[9] Spirent, "Spirent Campus Overview," 2017. [Online]. Available: https: //support.spirent.com/SpirentCSC/SC_knowledgeView?id=TRN10243 